\title{
Satisfação de Necessidades Psicológicas Básicas em Crianças e Adolescentes: Adaptação e Validação da ESNPBR
}

\author{
Fulfilment of Children's and Adolescents' Basic Psychological Needs: Adaptation \\ and Validation of the Basic Psychological Needs Satisfaction Scale
}

\author{
Francisco Simões* \& Madalena Alarcão \\ Universidade de Coimbra, Coimbra, Portugal
}

\begin{abstract}
Resumo
O objetivo deste trabalho foi adaptar e validar a Escala de Satisfação das Necessidades Psicológicas Básicas na Relação para crianças e adolescentes da população portuguesa. O estudo abrangeu 510 participantes, com idades compreendidas entre os 9 e os 16 anos. Os resultados indicam níveis moderados a elevados de consistência interna e um bom ajustamento das soluções fatoriais com dois e com três fatores. Todavia, a solução fatorial com dois fatores é aquela que apresenta melhores índices de ajustamento. Os resultados sustentam, ainda, hipóteses colocadas ao nível da validade convergente entre os fatores encontrados e outras variáveis relacionadas. Conclui-se que o instrumento, na sua versão atual, é uma medida geral adequada do grau de satisfação das necessidades psicológicas básicas na relação.

Palavras-chave: Avaliação psicológica, necessidades psicológicas básicas.
\end{abstract}

\begin{abstract}
The aim of this study was to adapt and validate the Basic Psychological Needs Satisfaction Scale (BPNS) for Portuguese children and adolescents. It involved 510 participants aged between 9 and 16 years old. The results show moderate to high levels of internal consistency and a good adjustment of factorial solution models with two and three factors in accordance with the correspondent theoretical framework. However, the factorial model with two factors is the one that presents better fit. The results also support hypotheses regarding convergent validity between the factors found and other related variables. In sum, the current version of the questionnaire seems to be an appropriate general measure of basic psychological needs in relationships.

Keywords: Psychological assessment, basic psychological needs.
\end{abstract}

A Teoria da Auto-Determinação (TAD) é uma macroteoria da motivação, da personalidade e do bem-estar. A sua premissa central é que a motivação pode oscilar num contínuo entre a desmotivação e a motivação intrínseca. Enquanto estado motivacional ótimo, a motivação intrínseca ocorre quando o sujeito desenvolve comportamentos autodeterminados, baseados num locus de controle interno e em processos regulatórios como o interesse genuíno e a satisfação (Niemec \& Ryan, 2009). Uma parte importante do trabalho desenvolvido pela TAD centra-se na análise das condições que poderão suscitar a motivação em diferentes contextos, como organizações (Baard, Deci, \& Ryan, 2004), saúde (Deci \& Ryan, 2008) ou educação (Niemec

\footnotetext{
* Endereço para correspondência: Universidade de Coimbra, Rua do Colégio Novo, Coimbra, Portugal 3001-802. E-mail: francisco.simoes@cipp-terceira.com e malarcao@uc.pt

Agradecimentos: Os autores desejam agradecer ao Prof. Cícero Roberto Pereira e aos revisores deste estudo pelos seus contributos. Este trabalho foi apoiado pela Fundação para a Ciência e para a Tecnologia (SRFH/ $\mathrm{BD} / 60823 / 2009$ ).
}

\& Ryan, 2009). Para a TAD, tais condições dependem da satisfação das chamadas necessidades psicológicas básicas. As mesmas são disposições de caráter inato, cuja satisfação serve de base à sobrevivência, ao crescimento e à integração do sujeito. De acordo com esta visão teórica, é possível distinguir três necessidades psicológicas básicas: relação, competência e autonomia (Deci \& Ryan, 2008; Ryan \& Deci, 2000). A necessidade de relação envolve a orientação pessoal para estabelecer laços relacionais fortes, estáveis e duradouros que promovam o sentido de aceitação e de compreensão por parte dos outros. Por sua vez, a necessidade de competência refere-se a um sentimento pessoal de eficácia, como consequência do sujeito desenvolver uma perceção de sucesso e capacidade, perante situações que se apresentam como desafiantes (Patrick, Knee, Canevello, \& Lonsbary, 2007). Finalmente a necessidade de autonomia pode manifestar-se em termos de capacidade para iniciar tarefas ou tomar decisões, controlo volitivo e assunção das consequências do próprio comportamento (La Guardia \& Patrick, 2008). Para a TAD, a conceptualização das necessidades psicológicas básicas só fica completa se as mesmas 
forem distinguidas dos desejos. Enquanto as primeiras se orientam para o desenvolvimento pessoal, estes últimos são motivos que também despoletam e orientam a ação humana, mas que produzem efeitos considerados danosos (Baard et al., 2004).

A satisfação das necessidades psicológicas básicas assenta na qualidade das relações interpessoais as quais são entendidas como sendo essencialmente dinâmicas. O seu dinamismo decorre do fato do comportamento humano variar em função de inúmeros aspetos tais como as pessoas com quem interagimos, os contextos em que o fazemos ou o estádio de desenvolvimento das diferentes relações interpessoais (La Guardia \& Patrick, 2008). Apesar dessa variabilidade, o extenso corpo de estudos empíricos parece sustentar que: (a) níveis elevados de satisfação das necessidades psicológicas básicas surgem, geralmente, associados a maior autodeterminação comportamental, motivação intrínseca e processos de regulação do comportamento, como o interesse genuíno ou a satisfação pessoal (Ryan \& Deci, 2000); (b) a associação entre níveis de satisfação mais elevados das necessidades de relação, competência e autonomia e maior grau de autodeterminação pessoal parece ser independente dos efeitos contextuais e culturais (Chirkov, Ryan, \& Wellnes 2005).

Diversos questionários têm sido desenvolvidos no quadro da TAD para avaliar globalmente o grau percebido de satisfação das necessidades psicológicas básicas, com alguns a permitirem a análise da satisfação de cada uma delas. Uma parte desses instrumentos tem vindo a ser traduzida das suas versões originais em inglês para a língua portuguesa, ao mesmo tempo que são estudadas as suas qualidades psicométricas. Todavia, verificou-se que não estava disponível, em português, um questionário adaptado e validado para crianças e adolescentes que analisasse esse conceito. De modo a responder a esta lacuna, o objetivo central do presente trabalho consiste em apresentar os resultados do processo de adaptação e validação da Escala de Satisfação de Necessidades Psicológicas Básicas na Relação (ESNPBR) para crianças e adolescentes da população portuguesa, com idades compreendidas entre os 9 e os 16 anos. Trata-se de um instrumento de carácter breve e flexível, pois permite avaliar a satisfação das necessidades psicológicas básicas em diversos contextos e na relação com diferentes pessoas. Estas particularidades da ESNPBR deram resposta a um segundo objetivo deste estudo, mormente a disponibilização de um instrumento de avaliação das necessidades psicológicas básicas ajustado às características da população em causa, nomeadamente pela sua brevidade (Moreira, 2004).

De maneira a concretizar as finalidades desta investigação, foi feita uma análise das qualidades psicométricas do instrumento ao nível da consistência interna dos resultados, da estrutura fatorial subjacente e da validade convergente. Os resultados desse trabalho, bem como algumas limitações encontradas e os procedimentos adotados com vista a ultrapassá-las, são apresentados e discutidos nos pontos seguintes.

\section{Método}

\section{Amostra}

A amostra foi recolhida em cinco escolas da rede de ensino público, da Região Autónoma dos Açores, em Portugal. Foram convidados a participar todos os alunos que frequentavam o $5^{\circ}$ e o $7^{\circ}$ anos de escolaridade, nos estabelecimentos de ensino envolvidos. Considerando os critérios de inclusão na amostra, foram selecionadas 27 turmas, com um total de 588 alunos inscritos. A amostra final ficou constituída por 510 alunos, o que se traduz numa taxa de participação de $86.7 \%$. Do número total de participantes, 238 (46,7\%) eram do sexo masculino e $272(53,3 \%)$ eram do sexo feminino. Quanto ao nível de escolaridade, 246 dos participantes frequentavam o $5^{\circ}$ ano, ao passo que 264 estavam inseridos em turmas do $7^{\circ}$ ano de escolaridade. A idade dos alunos variou entre os $9 \mathrm{e}$ os 16 anos $(M=11,12 ; D P=1,24)$.

\section{Instrumentos}

Escala de Satisfação das Necessidades Psicológicas Básicas na Relação (ESNPBR). A ESNPBR é constituída, na sua versão original, por nove itens, que podem ser classificados de 1 (Nada verdadeiro) a 7 (Completamente verdadeiro). Os itens estão agrupados em três fatores que correspondem ao suporte das necessidades psicológicas básicas definidas pela TAD (Relação, Competência e Autonomia). Todos os itens estão formulados de modo afirmativo, sendo que três deles são cotados em sentido inverso pois, nos níveis mais elevados, descrevem a não-satisfação da necessidade psicológica básica a que se reportam (e.g. Quando estou com elelela, sinto-me incapaz). A ESNPBR permite que o aplicador selecione, previamente, a relação sobre a qual pretende fazer a avaliação da satisfação das necessidades psicológicas básicas. O instrumento possibilita, assim, fazer uma avaliação de diferentes tipos de relações diversas como amizades, ligações com adultos de referência ou relações íntimas. Neste trabalho, a ESNPBR foi usada para que os participantes analisassem o grau de satisfação das suas necessidades básicas na relação com os seus professores com a função de diretores de turma. Os trabalhos em que a ESNPBR tem sido utilizada, na sua versão original, revelam uma consistência interna adequada, com os valores de alpha de Cronbach a variarem entre 0,85 e 0,94 (La Guardia, Ryan, Couchman, \& Deci, 2000).

Escala de Competência Percebida na Aprendizagem. A Escala de Competência Percebida na Aprendizagem (ECPA) é constituída por quatro itens, que permitem avaliar o grau subjetivo de competência dos sujeitos, num determinado contexto de aprendizagem. O instrumento, na sua versão original, em Língua Inglesa, permite ao sujeito analisar o seu grau de concordância em relação a cada uma das afirmações, de acordo com uma escala de resposta de sete pontos (de completamente falso a completamente verdadeiro). $\mathrm{Na}$ sua versão adaptada para crianças e adolescentes da população portuguesa, cada um dos itens (e.g. Vou ser capaz de atingir os meus objetivos, neste ano 
Simões, F. \& Alarcão, M. (2013). Satisfação de Necessidades Psicológicas Básicas em Crianças e Adolescentes: Adaptação e Validação da ESNPBR.

letivo) é avaliado através do recurso a uma escala ordinal de cinco pontos, que varia entre 1 (nunca) e 5 (sempre). A versão para crianças e adolescentes, adaptada para a população portuguesa, apresenta um alpha de Cronbach de 0,85 (Simões \& Alarcão, 2011).

Escala de Escolha Percebida na Aprendizagem. A Escala de Escolha Percebida na Aprendizagem (EEPA) é, originalmente, uma subescala da Escala de Auto-Determinação (Sheldon, 1995). A mesma foi adaptada a partir da versão traduzida para a população portuguesa (Silva et al., 2007) e avalia em que medida os sujeitos consideram as suas decisões e escolhas na aprendizagem como resultado da sua própria iniciativa. A escala é constituída por 5 itens formulados de modo afirmativo (e.g. Sinto que sou eu a escolher aquilo que faço), em relação às quais os sujeitos deverão posicionar-se de acordo com uma escala ordinal de 1 (nunca) a 5 (sempre). Diversos estudos realizados com a versão original revelam valores de alpha de Cronbach que variam entre 0,86 e 0,92 (Sheldon, 1995). A versão para crianças e adolescentes, adaptada para a população Portuguesa, apresenta um alpha de Cronbach de 0,80 (Simões \& Alarcão, 2011).

Escala de Esperança para Crianças. A Escala de Esperança para Crianças (EEC) foi desenvolvida por Snyder et al. (1997) para avaliar os pensamentos de esperança relacionados com objetivos, em crianças e adolescentes com idades entre os 8 e os 16 anos. A escala é constituída por 6 itens, formulados de modo afirmativo, sendo que 3 deles avaliam a componente denominado "Iniciativa" (e.g. Penso que estou a fazer bem as coisas) e os restantes 3 itens avaliam a componente designada por "Caminhos" (e.g. Consigo pensar em muitas maneiras de conseguir as coisas que considero importantes). Os itens que avaliam cada uma das componentes são apresentados de forma alternada e podem ser classificados de acordo com uma escala ordinal de seis pontos entre 1 (nenhuma das vezes) e 6 (todas as vezes). Estudos realizados pelos autores da versão original revelam uma consistência interna com valores que variaram entre 0,72 e 0,86 e um coeficiente de fidelidade teste-reteste com valores que oscilam entre 0,71 e 0,73 (Snyder et al., 1997). Um alpha de Cronbach de 0,81 foi encontrado para a versão portuguesa do instrumento (Marques, Pais-Ribeiro, \& Lopez, 2008).

Kidscreen-27. O Kidscreen-27 é um instrumento de avaliação da perceção da qualidade de vida relacionada com a saúde, em crianças e adolescentes saudáveis ou com situações de doença crónica, com idades compreendidas entre os 8 e os 18 anos. Trata-se de um questionário de autopreenchimento em que os sujeitos são convidados a posicionar-se perante os itens, considerando como correu a última semana. O mesmo é constituído por cinco subescalas: Bem-Estar Físico, Bem-Estar Psicológico, Autonomia, Suporte Social e Grupo de Pares e Ambiente Escolar (Ravens-Sieberer et al., 2007). Para cada um dos itens, os sujeitos possuem 5 hipóteses de resposta. Consoante o item em causa, as escalas apresentadas são de intensidade (variando entre nada e extremamente) ou de frequência (oscilando entre nunca e sempre). O Kidscreen-27 apresenta valores de alpha de Cronbach que variam entre 0,80 e 0,84 para as diferentes subescalas que a constituem (Ravens-Sieberer et al., 2007). A versão portuguesa (Gaspar \& Matos, 2008) tem sido utilizada em contexto de investigação, com níveis de consistência interna moderados a elevados para a totalidade da escala $(\alpha=0,89)$ (Simões \& Alarcão, 2011).

\section{Procedimentos e Análise dos Dados}

A realização do presente estudo foi antecedida de um pedido de autorização aos autores para utilização e adaptação dos instrumentos. A versão original da ESNPBR foi traduzida da sua versão original em inglês para a língua portuguesa por duas pessoas formadas em psicologia e fluentes em ambas as línguas. Seguiu-se um processo de retroversão, realizado por outras duas pessoas, também fluentes nos dois idiomas, sem acesso à versão original do instrumento, de modo a favorecer a identificação de discrepâncias entre ambas formulações. De maneira a garantir um equilíbrio entre a definição teórica de necessidades psicológicas básicas, tal como é proposta na literatura pela TAD, e o pressuposto de tornar a linguagem acessível a crianças e adolescentes, o processo de tradução e retroversão foi triangulado com a opinião de dois docentes formados, simultaneamente, em português e inglês. Seguiu-se um estudo-piloto em que 11 crianças e adolescentes, com idades compreendidas entre os 10 e os 16 anos, procederam ao preenchimento individual de todo o protocolo do estudo, acompanhado de uma discussão sobre o mesmo. A triangulação da informação com docentes bem como a discussão item a item realizada com os sujeitos que participaram no estudo-piloto conduziu a ajustamentos em sete itens. As alterações introduzidas foram feitas ao nível da estrutura frásica dos itens, substituição de palavras por sinónimos mais inteligíveis ou emprego de diferentes tempos verbais.

Depois de concluído o processo de adaptação dos instrumentos, foi solicitada autorização à Direção Regional da Educação e Formação do Governo Regional dos Açores para a administração do protocolo do estudo em escolas da rede pública de ensino da região. Obtido esse consentimento oficial, foi feito um pedido de colaboração a cinco escolas, através de contacto com os respetivos conselhos executivos, as quais já colaboravam com os investigadores noutro estudo. Uma vez garantida a cooperação das escolas, foram contactados os representantes legais dos alunos, através de documento escrito enviado pelo diretor de turma. Nele eram descritos os objetivos e procedimentos da investigação a realizar, ao mesmo tempo que se solicitava a autorização para que os alunos pelos quais eram responsáveis participassem no mesmo. No momento da aplicação dos instrumentos os participantes foram sempre questionados sobre a sua disponibilidade para colaborarem.

A aplicação dos instrumentos foi coletiva, tendo sido realizada por turma, numa aula da disciplina de Cidadania, de acordo com o estipulado pelos conselhos executivos 
das escolas. A dimensão dos grupos que participaram no estudo variou entre 10 e 25 alunos. A administração dos instrumentos foi feita pelo primeiro autor, de acordo com um guião de procedimentos criado para o efeito, não estando presente qualquer docente na sala no momento da aplicação. Antes de preencherem os instrumentos, os participantes foram elucidados sobre o modo de organização do protocolo, as questões de confidencialidade das respostas e a importância de todas as perguntas serem devidamente respondidas.

Os dados foram analisados com recurso ao PASW Statistics 18 . Foram avaliados quatro aspetos complementares. A análise exploratória dos dados começou por revelar que a percentagem de dados omissos no preenchimento da ESNPBR foi baixa (0,77\%). Mesmo assim, foi feita a sua estimação e substituição, com recurso ao método de cálculo do valor médio da série (series mean), disponível no programa informático usado. Seguidamente, foi conduzida uma Análise em Componentes Principais sobre a matriz de correlações de Spearman. Foi, depois, realizada uma avaliação da consistência interna dos instrumentos, através do cálculo do alpha de Cronbach para a totalidade do questionário e para cada uma das componentes extraídas. Este parâmetro foi ainda estudado na contingência de eliminação de cada um dos itens (scale if item-deleted) que o constituem. Este procedimento foi sucedido de uma análise fatorial confirmatória realizada no programa Amos 18 e que se destinou a aumentar a compreensibilidade do modelo. Foram testados, de forma comparativa, modelos com dois e três fatores, atendendo aos índices de aderência (Goodness-of-fit). Posteriormente, foi analisada a validade convergente, através do cálculo de correlações de Spear- man entre todos os fatores dos diferentes questionários integrados na bateria deste estudo.

\section{Resultados}

\section{Análise Exploratória dos Dados}

As estatísticas descritivas foram $M=31,10$ e $D P=7,09$, para a totalidade da ESNPBR. Quanto à assimetria dos dados, foi observado que os diferentes itens da ESNPBR possuem uma distribuição tendencialmente assimétrica, cujos valores variam entre $-1,018$ para o item 2 e $-0,069$ para o item $9(D P=0,108)$. Já os valores de achatamento oscilaram entre -1,564 para o item 9 e $-0,185$ para o item $3(D P=0,216)$. Apenas os itens 4,6 e 9 apresentaram um valor de assimetria entre $-0,5$ e 0,5 e os itens 1 e 3 revelaram um valor de achatamento dentro do referencial indicativo de uma distribuição próxima da normalidade (Maroco, 2007).

\section{Análise em Componentes Principais}

O estudo da estrutura relacional das variáveis do ESNPBR foi feito através da realização de uma Análise em Componentes Principais (ACP). Os componentes retidos foram aqueles que apresentavam um eigenvalue superior a 1 , em consonância com o scree plot $\mathrm{e}$ a percentagem de variância retida (Maroco, 2007). De modo a definir as componentes a reter, foi adotado o método de Kaiser e rotação oblíqua. De acordo com estes princípios de análise, foi possível resumir a informação relacional entre as variáveis em duas componentes que explicam $70,26 \%$ da variância total das variáveis originais. A Tabela 1 apresenta uma síntese dos resultados encontrados nesta análise.

Tabela 1

Componentes Principais extraídas do Questionário de Satisfação de Necessidades Psicológicas Básicas na Relação (loadings, eigenvalues e percentagem de variância explicada)

\begin{tabular}{lcc}
\hline Item & Componente 1 & Componente 2 \\
\hline Quando estou com ele/ela, sinto que posso ser eu próprio/a. & 0,77 & \\
Quando estou com ele/ela, sinto-me capaz de fazer as coisas bem. & 0,83 & \\
Quando estou com ele/ela sinto que se interessa e que gosta de mim. & 0,80 & \\
Quando estou com ele/ela, sinto que posso dizer o que penso. & 0,75 & \\
Quando estou com ele/ela, sinto que sou capaz de fazer as coisas & 0,80 & \\
depressa e bem. & 0,86 & 0,89 \\
Quando estou com ele/ela, sinto que se interessa e que gosta de mim. & & 0,91 \\
Quando estou com ele/ela, sinto-me incapaz. & & 0,87 \\
Quando estou com ele/ela, sinto um grande afastamento entre nós. & \\
Quando estou com ele/ela, sinto-me obrigado a ser de uma maneira & \\
que não sou. & 3,904 & 2,420 \\
Eigenvalue & $43,38 \%$ & $26,88 \%$ \\
\% de variância explicada & 0,90 & 0,88 \\
Alpha de Cronbach &
\end{tabular}


Simões, F. \& Alarcão, M. (2013). Satisfação de Necessidades Psicológicas Básicas em Crianças e Adolescentes: Adaptação e Validação da ESNPBR.

Em consonância com os pesos de cada variável em cada componente, é possível indicar uma primeira componente com um eigenvalue de 3,904, responsável por $43,38 \%$ da variância total explicada. Esta aglutinou as 6 variáveis codificadas em ordem direta, podendo ser nomeada como satisfação das necessidades psicológicas básicas na relação. Uma segunda componente apresentou um eigenvalue de 2,42 , explicando $26,88 \%$ da variabilidade total. A mesma agregou as 3 variáveis codificadas em sentido inverso, pelo que foi denominada como não-satisfação das necessidades psicológicas básicas. $\mathrm{O}$ teste de esfericidade de Bartlett apresenta um valor de $R=2426,70(p=0,000)$ e o teste de Kaiser-Meyer-Olkin de $R=0,84$. Ambos os valores confirmam a adequação do uso do instrumento nesta amostra.

\section{Consistência Interna}

A análise da consistência interna revelou um alpha de Cronbach de 0,80 para a totalidade do questionário, o qual pode ser considerado moderado a elevado (Revelle \& Zinbarg, 2009). A análise da consistência interna, com base no critério if item-deleted, evidenciou que a remoção de qualquer um dos itens que constituíam o instrumento analisado não resultou num acréscimo do valor global do valor de alpha de Cronbach, mas antes na sua diminuição.

\section{Análise Fatorial Confirmatória}

Após ter sido realizada a ACP, foi conduzida uma Análise Fatorial Confirmatória (AFC), com recurso ao programa informático Amos 18. Este procedimento visou, por um lado, verificar a adequação de uma estrutura fatorial organizada em três fatores, em consonância com o instrumento original e, por outro, compará-la com um modelo com apenas dois fatores sugerido pela ACP, no que se refere à qualidade do seu ajustamento.

Segundo os resultados da AFC apresentados na Tabela 2, o modelo fatorial que evidencia melhor ajustamento é aquele que organiza os itens da ESNPBR em dois fatores. De acordo com esta estrutura, o primeiro fator integrou os itens $1,2,3,5,7$ e 8 , enquanto o segundo fator abarcou os itens $4,6 \mathrm{e} 9$. O modelo com dois fatores começa por revelar um Qui-quadrado de 60,885, com 27 graus de liberdade $(g l)$ e CMIN/DF de 2,899. Este valor indicia um ajustamento adequado do modelo à amostra, de acordo com o critério de que o valor CMIN/DF deverá oscilar entre 2 e 5 (Marsh \& Hocevar, 1985). De modo complementar, foram calculados os índices de aderência (Goodness-of-fit). O Incremental Fit Index (IFI) apresentou um valor de ajustamento de 0,956 , enquanto o valor de ajustamento do Normed Fit Index (NFI) se situou nos 0,923. Por sua vez, o Compared Fit Index (CFI) apresentou um valor de 0,955. Estes dados apontam, igualmente, para um bom ajustamento do modelo fatorial testado, visto que este é considerado adequado para qualquer um destes índices quando se registam valores iguais ou superiores a 0,90 (Bentler \& Dundgeon, 1996). De igual modo, foi feita, também, a análise do Root Mean Square Error of Approximation (RMSEA), o qual apresentou um valor de 0,06 . Apesar de bastante afastado de zero, autores como Arbuckle, (2009) referem que valores iguais ou inferiores a 0,08 podem ser considerados aceitáveis. Por sua vez, o modelo estruturado em três fatores apresenta um Qui-quadrado de 85,791, com 21 graus de liberdade $(g l)$ e CMIN/DF de 4,085, ambos mais elevados do que sucede no modelo com dois fatores. Relativamente aos índices de aderência, o modelo com três fatores evidencia valores ligeiramente melhores: o IFI corresponde a 0,975 , enquanto o NFI se situa nos 0,967 , ao passo que o CFI é de 0,975. Ainda por comparação ao modelo com dois fatores, o valor de RMSEA do modelo com três fatores é superior, situando-se em 0,08. Em suma, embora os índices de ajustamento de ambos os modelos sejam aceitáveis, o modelo com dois fatores regista um melhor ajustamento, de resto documentado pelo valor de delta qui-quadrado da diferença entre os modelos testados $(\delta=24,906)$. Perante estes resultados, e em consonância com a TAD, poder-se-á afirmar que o Fator 1 surge associado à Satisfação das Necessidades Psicológicas Básicas na Relação (SNPBR) e o Fator 2 à Não-Satisfação de Necessidades Psicológicas Básicas (NSNPBR).

Tabela 2

Índices de Adequação dos Modelos Fatoriais Testados

\begin{tabular}{lcc}
\hline Índice de ajustamento & Modelo 2 fatores & Modelo 3 fatores \\
\hline Qui-quadrado* & $60,885(g l=27)$ & $85,791(g l=21)$ \\
CMIN/DF & 4,328 & 4,085 \\
IFI & 0,956 & 0,975 \\
NFI & 0,923 & 0,967 \\
CFI & 0,955 & 0,974 \\
RMSEA & 0,060 & 0,080 \\
\hline
\end{tabular}

Nota. $* \delta=24,906$ 
Psicologia: Reflexão e Crítica, 26(2), 261-269.

Tabela 3

Resultados da Avaliação da Validade Convergente entre as Subescalas da ESNPBR e os Fatores dos Instrumentos Usados no Estudo

\begin{tabular}{lcc}
\hline & SNPBR & NSNPBR \\
\hline Competência percebida na aprendizagem & $0,46^{* *}$ & $-0,03$ \\
Escolha percebida na aprendizagem & $0,21^{* *}$ & 0,03 \\
Esperança/iniciativa & $0,50^{* *}$ & $-0,04$ \\
Esperança/caminhos & $0,43^{* *}$ & 0,004 \\
Contexto escolar (K-27) & $0,52^{* *}$ & $-0,07$ \\
Bem-estar físico (K-27) & $0,33^{* *}$ & $-0,06$ \\
Bem-estar psicológico (K-27) & $0,39^{* *}$ & $-0,07$ \\
Autonomia e relação parental (K-27) & $0,35^{* *}$ & 0,03 \\
Suporte social e grupo de pares K-27) & $0,34^{* *}$ & 0,002 \\
\hline
\end{tabular}

Nota. $* * p<0,001$.

\section{Validade Convergente}

Ao nível do estudo da validade convergente, foi colocada a hipótese de que o fator que representa a Satisfação das Necessidades Psicológicas na Relação estaria positiva e significativamente correlacionado com os fatores inerentes aos restantes instrumentos utilizados. A hipótese inversa foi avançada no caso do fator que representa a Não-Satisfação das Necessidades Psicológicas Básicas na Relação. Foram consideradas de interesse as correlações de Spearman significativas, com valores acima de 0,30 (Maroco, 2007).

Uma leitura mais pormenorizada da Tabela 3 permite verificar que todas as correlações esperadas ao nível da validade convergente entre o fator Satisfação das Necessidades Psicológicas Básicas na Relação e os fatores inerentes aos instrumentos aplicados são moderadamente significativas $(\rho \mathrm{s}>0,30)$, com exceção da correlação entre este fator e a EEPA $(\rho \mathrm{s}=0,21)$. De entre as restantes correlações, a mais baixa ocorre entre a Satisfação de Necessidades Psicológicas na Relação e a subescala Bem-Estar Físico, do Kidscreen-27, $(\rho \mathrm{s}=0,33)$. Já a mais elevada é observada entre esse mesmo fator do instrumento sob estudo e outra subescala do Kidscreen-27, a subescala Ambiente Escolar $(\rho s=0,52)$. Também em consonância com as hipóteses colocadas quanto ao estudo da validade convergente, foram encontradas várias correlações negativas e significativas entre o fator Não-Satisfação das Necessidades Psicológicas Básicas e outras das escalas utilizadas neste trabalho. Tais correlações são, porém, de pequena dimensão. A mais baixa é registada entre este fator do instrumento estudado e a Competência Percebida na Aprendizagem $(\rho s=-0,03)$, ao passo que a mais alta ocorre com as subescalas de Ambiente Escolar e Bem-Estar Psicológico $(\rho s=-0,07)$. As restantes correlações testadas em relação ao fator Não-Satisfação das Necessidades Psicológicas Básicas são fracas e positivas, mas não significativas.

\section{Discussão dos Resultados}

Muitas das investigações que têm procurado compreender o suporte relacional dado às necessidades psicológicas básicas apoiam-se em instrumentos de recolha de dados inspirados nos pressupostos da TAD. Esses instrumentos, com particular destaque para os questionários, avaliam a satisfação de necessidades psicológicas básicas, com uma estrutura de fatores correspondente às necessidades de relação, competência e autonomia que são propostas pela mesma teoria. O presente trabalho teve por objetivo a adaptação e validação de um questionário baseado nesse modelo teórico, a ESNPBR, procurando disponibilizar uma versão do mesmo para crianças e adolescentes portugueses. As suas propriedades psicométricas foram estudadas no quadro das relações pedagógicas dos participantes com os respetivos diretores de turma.

A estrutura do instrumento revelada pela $\mathrm{ACP}$ apontou para a existência de duas componentes na ESNPBR. Uma primeira agregou as variáveis codificadas em sentido direto, ao passo que a segunda agrupou as variáveis codificadas em sentido inverso. Do ponto de vista da consistência interna, a ESNPBR apresenta valores de fiabilidade moderados a elevados ( $\alpha=0,80$; Revelle \& Zinbarg, 2009), com um contributo equilibrado e relevante dos diferentes itens para a consistência interna do instrumento, de acordo com o critério de análise if item deleted. A estrutura dicotómica decorrente da ACP foi testada e comparada com um modelo de três fatores semelhante ao proposto no instrumento original em língua inglesa, o qual organiza os itens de acordo com as três necessidades psicológicas básicas defendidas pela TAD. Através da AFC, foi possível verificar que ambos os modelos evidenciam um ajustamento aceitável. Todavia, a diferença do delta qui-quadrado entre os modelos estudados $(\delta=24,906)$ indicia que o modelo com dois fatores denota um melhor ajustamento do que o modelo com três fatores. Assim sendo, interessa discutir porque 
Simões, F. \& Alarcão, M. (2013). Satisfação de Necessidades Psicológicas Básicas em Crianças e Adolescentes: Adaptação e Validação da ESNPBR.

é que a estrutura de três fatores sustentada teoricamente pela TAD, embora seja aceitável, não se apresenta como o modelo mais adequado, neste estudo. A interpretação de que as necessidades psicológicas básicas, tal como são postuladas pela TAD, não apresentarão validade cultural para a população portuguesa poderia ser uma das hipóteses explicativas avançadas. Todavia, no presente trabalho, essa análise interpretativa poderá ser não apenas limitada como arriscada. Estudos anteriores têm sustentado que tanto as necessidades psicológicas em si mesmas, como a estrutura sugerida pela TAD, são inatas ao ser humano, não parecendo ser influenciadas nem pelos contextos de vida, nem pela cultura (Chirkov et al., 2005; Jang, Reeve, Ryan, \& Kim, 2009). Em paralelo, é conveniente sublinhar que quer uma organização mais simples das necessidades psicológicas básicas, estruturada em apenas dois fatores, quer um modelo mais complexo, que distingue claramente as necessidades de relação, competência e autonomia, têm sido encontrados em pesquisas anteriores. No caso específico das estruturas dicotómicas, estas parecem descrever, sobretudo, o nível de satisfação percebido pelos sujeitos num dado contexto que oscila entre a sua não-satisfação e a sua satisfação (Sheldon, 1995). Segundo Van den Broeck, Vansteenkiste, De Witte, Soenens, e Lens (2010), tal pode ser atribuído a uma organização teórica superior que será anterior a uma conceptualização mais elaborada como aquela que corresponde às próprias necessidades psicológicas básicas.

Em alternativa à possível influência cultural, outras hipóteses poderão ser mais elucidativas relativamente ao melhor ajustamento encontrado para o modelo com dois fatores na versão portuguesa da ESNPBR. Neste quadro, assumem particular relevo algumas opções metodológicas que foram tomadas. Desde logo, foi mantido o número reduzido de itens, por se entender que o carácter breve do instrumento era pertinente, tendo em vista contextos de investigação e de intervenção com crianças e adolescentes. Esse número encontra-se num limite teoricamente considerado mínimo de três itens para cada fator (Moreira, 2004). Acrescenta-se a esta decisão uma segunda que consistiu na alteração da escala de resposta, passando a mesma de sete para cinco pontos. Embora essa solução tenha sido motivada por uma necessidade de melhor adaptação do instrumento à capacidade de discriminação dos sujeitos notória no estudo-piloto, a mesma poderá ter diminuído a possibilidade do instrumento diferenciar, com maior clareza, fatores correspondentes às necessidades de relação, competência e autonomia. Será de salientar, ainda, como os resultados encontrados poderão ser consequência da interação do instrumento com as características dos próprios participantes. A ESNPBR tem sido utilizada, na sua versão original, para a avaliar a satisfação das necessidades psicológicas básicas em relações entre adultos. Possivelmente, no caso de crianças e adolescentes, faz sentido alargar o número de itens teoricamente relacionados com as necessidades de relação, competência e autonomia, favorecendo assim uma maior diferenciação das mesmas e, consequentemente, uma melhoria do ajustamento já observado para o modelo com três fatores. Esse alargamento dos itens integrados na ESNPBR não deve, contudo, ser feito de tal forma que comprometa o carácter breve e, por isso, útil deste instrumento, nomeadamente em contextos de investigação.

O presente estudo envolveu, também, aferição da validade convergente da ESNPBR. As hipóteses colocadas a este nível foram, genericamente, corroboradas pelos resultados encontrados. Em concreto, foi possível observar que com exceção da EEPA ( $\rho$ s $>0,21)$, o fator de Satisfação das Necessidades Psicológicas Básicas na Relação da ESNPBR apresenta correlações positivas, significativas e moderadas $(\rho \mathrm{s}>0,30)$ com todos os restantes fatores que fazem parte dos instrumentos utilizados. Pelo contrário, o fator de Não-Satisfação das Necessidades Psicológicas Básicas na Relação da ESNPBR tem diversas correlações negativas, mas não-significativas, com diversos dos fatores que fizeram parte do protocolo deste estudo. Tratam-se de correlações fracas $(\rho \mathbf{s}>0,30)$ e algumas delas são positivas. Estes resultados são consistentes com evidências de outras pesquisas, as quais demonstram que uma maior satisfação das necessidades básicas está associada a uma melhor adaptação em dimensões específicas ponderadas neste trabalho, tais como o bem-estar psicológico (Vérroneau, Koestner, \& Abela, 2004), a competência percebida (Niemec \& Ryan, 2009) ou a adaptação à escola (Milyavskaya et al., 2009).

É importante referir que algumas limitações poderão ter, também, concorrido para os vários resultados apurados. Merece especial relevo a possível interferência de variáveis contextuais. A normalização das condições de aplicação dos instrumentos, através da elaboração de um guião de procedimentos detalhado, procurou responder a esse possível desafio. Todavia, pareceu haver alguma dificuldade dos participantes em estabelecer uma distinção entre o processo de investigação e as atividades de aprendizagem formal, bem como entre o próprio investigador e os professores. Dúvidas pontuais sobre a confidencialidade dos dados, veiculadas durante as sessões de aplicação dos instrumentos, servem para ilustrar o potencial impacto deste fator nas respostas dadas. Não menos importante é o fato de se ter assistido, nos últimos anos, a uma massificação de processos de investigação por questionário em meio escolar, uma contingência que poderá ter, igualmente, condicionado a motivação e a atenção dos participantes no preenchimento do protocolo (Strange, Forest, \& Oakley, 2003).

De modo complementar, e em jeito de síntese, algumas recomendações parecem ser pertinentes no intuito de diversificar o conhecimento sobre as propriedades psicométricas deste questionário. De entre todas, a mais pertinente parece ser a já referenciada inclusão de novos itens no questionário, a par de outras, tais como: (a) uma análise diferencial e comparativa do comportamento da ESNPBR, em função do seu uso relativamente a diferentes relações (pais, 
amizades, entre outras) e de algumas variáveis pessoais (género e idade); (b) a realização de estudos com baterias de instrumentos para investigação inteiramente baseadas na TAD, desde que os mesmos se encontrem disponíveis; e (c) avaliações sobre a estabilidade temporal da ESNPBR, com diferentes intervalos temporais entre a primeira e a segunda aplicações do instrumento.

\section{Conclusão}

Neste estudo, procurou aferir-se as qualidades psicométricas da ESNPBR, na sua versão traduzida e adaptada para crianças e adolescentes da população portuguesa. Os dados indicam níveis de consistência interna moderados a elevados e corroboram que a solução fatorial com dois fatores, testada através do recurso à $\mathrm{AFC}$, é aquela que apresenta melhores indicadores de ajustamento. O reduzido número de itens da ESNPBR e a redução da escala de resposta usada, por comparação com o instrumento original, poderão justificar o fato do modelo fatorial correspondente às três necessidades psicológicas básicas não ser aquele que reúne melhores indicadores de ajustamento. Mesmo assim, essa solução fatorial apresenta um ajustamento aceitável, pelo que a realização de novos estudos sobre este instrumento, integrando um maior número de itens, se apresenta como recomendável e promissor. Assim, conclui-se, que o instrumento, na sua versão atual, se constitui como uma medida geral adequada do grau de satisfação das necessidades psicológicas básicas na relação.

\section{Referências}

Arbuckle, J. L. (2009). Amos 18 User's Guide. Chicago, IL: Statistical Package for the Social Sciences.

Baard, P. P., Deci, E. L., \& Ryan, R. M. (2004). Intrinsic need satisfaction: A motivational basis of performance and wellbeing in two work settings. Journal of Applied Social Psychology, 34(10), 2045-2068.

Bentler, P., \& Dudgeon, P. (1996). Covariance structure analysis: Statistical practice, theory, and directions. Annual Review of Psychology, 47, 563-655.

Chirkov, V. I., Ryan, R. M., \& Willness, C. (2005). Cultural context and psychological needs in Canada and Brazil: Testing a self-determination approach to the internalization of cultural practices, identity, and well-being. Journal of Cross-Cultural Psychology, 36, 423-443.

Deci, E. L., \& Ryan, R. M. (2008). Self-determination Theory: A macrotheory of human motivation, development and health. Canadian Psychology, 29(3), 201-209, 182-185.

Gaspar, T., \& Matos, M. G. (2008). Qualidade de vida em crianças e adolescentes: Versão portuguesa dos instrumentos KIDSCREEN-52. Cruz Quebrada, Portugal: Aventura Social e Saúde.

Jang, H., Reeve, J., Ryan, R. M., \& Kim, A. (2009). Can Selfdetermination Theory explain what underlies the productive, satisfying learning experiences of collectivistically oriented Korean students? Journal of Educational Psychology, 101(3), 644-661.
La Guardia, J. G., \& Patrick, H. (2008). Self-determination Theory as a fundamental theory of close relationships. $\mathrm{Ca}$ nadian Psychology, 29(3), 201-209.

La Guardia, J. G., Ryan, R. M., Couchman, C. E., \& Deci, E. L. (2000). Within-person variation in security of attachment: A Self-determination Theory perspective on attachment, need fulfillment, and well-being. Journal of Personality and Social Psychology, 79(3), 367-384.

Maroco, J. (2007). Análise estatística com utilização de SPSS. Lisboa, Portugal: Edições Sílabo.

Marques, S. C., Pais-Ribeiro, J. L., \& Lopez, S. (2008). Estabilidade temporal das Escalas de Esperança para Crianças e de Satisfação com a Vida para Estudantes. Psicologia, Saúde e Doenças, 9(2), 245-252.

Marsh, H. W., \& Hocevar, D. (1985). Application of confirmatory fator analysis to the study of self-concept: First- and higher-order fator models and their invariance across groups. Psychological Bulletin, 97, 562-582.

Milyavskaya, M., Gingras, I., Mageau, G. A., Koestner, R., Gagnon, H., Fang, J. Q., \& Boiché, J. (2009). Balance across contexts: Importance of balanced need satisfaction across various life domains. Personality and Social Psychology Bulletin, 35(8), 1031-1045. doi: 10.1177/0146167209337036.

Moreira, J. (2004). Questionários: Teoria e prática. Coimbra, Portugal: Almedina.

Niemec, C. P., \& Ryan, R. M. (2009). Autonomy, competence and relatedness in the classroom: Applying self-determination to educational practice. Theory and Research in Education, 7(2), 133-134. doi: 10.1177/1477878509104318

Patrick, H., Knee, C. R., Canevello, A., \& Lonsbary, C. (2007). The role of need fulfillment in relationship functioning and well-being: A Self-determination Theory perspective. Journal of Personality and Social Psychology, 92(3), 434-457.

Ravens-Sieberer, U., Auquier, P., Erhart, M., Gosch, A., Rajmil, L., Bruil, J., ...The European KIDSCREEN Group. (2007). The KIDSCREEN-27 quality of life measure for children and adolescents: Psychometric results of a cross-cultural survey in 13 European countries. Quality of Life Reearch, $16,1347-1356$.

Revelle, W., \& Zinbarg, R. (2009). Coefficients alpha, beta, omega, and the glb: Comments on Sijtsma. Psychometrika, 74(1), 145-154.

Ryan, R. M., \& Deci, E. L. (2000). Self-determination Theory and the facilitation of intrinsic motivation, social development, and well-being. American Psychologist, 55(1), 68-78.

Sheldon, K. M. (1995). Creativity and self-determination in personality. Creative Research Journal, 8(1), 25-36.

Silva, M. N., Vieira, P. N., Minderico, C. S., Castro, M. M., Coutinho, S. C., Santos, T. C., \& Teixeira, P. J. (2007). Testing a Portuguese Self-determination Theory-based psychometric battery. Poster presented at the Annual Meeting of International Society for Behavioral Nutrition and Physical Activity, Oslo, Norway.

Simões, F., \& Alarcão, M. (2011). Avaliação da motivação intrínseca na aprendizagem: Validação de duas escalas para crianças e adolescentes. Psico-USF, 16(3), 265-273.

Snyder, C. R., Hoza, B., Pelham, W. E., Rapoff, M., Ware, L., Danovsky, M., ...Stahl, K. J. (1997). The development and validation of the Children's Hope Scale. Journal of Pediatric Psychology, 22, 399-421.

Strange, V, Forest, S., \& Oakley A. (2003). Using research questionnaires with young people in schools: The influence of the school context. International Journal of Social Research Methodology, 6(4), 337-346. 
Simões, F. \& Alarcão, M. (2013). Satisfação de Necessidades Psicológicas Básicas em Crianças e Adolescentes: Adaptação e Validação da ESNPBR.

Van den Broeck, A., Vansteenkiste, M., De Witte, H., Soenens, B., \& Lens, W. (2010). Capturing autonomy, competence, and relatedness at work: Construction and initial validation of the Work-related Basic Need Satisfaction Scale. Journal of Occupational and Organizational Psychology, 83, 981-1002. doi: 10.1348/096317909X481382

Vérroneau, M., Koestner, R. F., \& Abela, J. R. Z. (2004). Intrinsic need satisfaction and well-being in children and adolescents: An application of Self-determination Theory. Journal of Social and Clinical Psychology, 24(2), 280-292. 